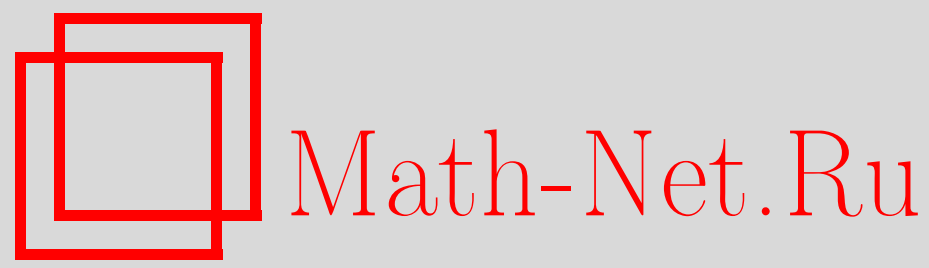

В. Т. Фоменко, Об одном свойстве конформных бесконечно малых деформаций многомерных поверхностей в римановом пространстве, Матем. заметки, 1996, том 59, выпуск 2, 284-290

DOI: https://doi.org/10.4213/mzm1715

Использование Общероссийского математического портала MathNet.Ru подразумевает, что вы прочитали и согласны с пользовательским соглашением

http://www . mathnet.ru/rus/agreement

Параметры загрузки:

IP : 54.164 .48 .24

26 апреля 2023 г., 03:27:34

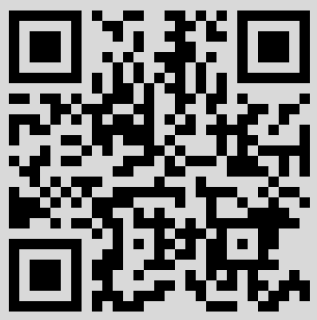




\section{ОБ ОДНОМ СВОЙСТВЕ КОНФОРМНЫХ БЕСКОНЕЧНО МАЛЫХ ДЕФОРМАЦИЙ МНОГОМЕРНЫХ ПОВЕРХНОСТЕЙ В РИМАНОВОМ ПРОСТРАНСТВЕ}

\section{В. Т. Фоменко}

1. Пусть $F^{k}-k$-мерная поверхность класса $C^{3}$ в $n$-мерном римановом пространстве $\mathbb{R}^{n}$ с метрической формой $a_{\alpha \beta} d y^{\alpha} d y^{\beta}$, где $a_{\alpha \beta} \in C^{3}$. Будем считать, что в окрестности каждой своей точки поверхность $F^{k}$ задается уравнениями

$$
y^{\alpha}=f^{\alpha}\left(x^{1}, x^{2}, \ldots, x^{k}\right),
$$

где $\left(x^{1}, x^{2}, \ldots, x^{k}\right)$ пробегает некоторую область $k$-мерного арифметического пространства.

Рассмотрим деформацию $\left\{F_{\varepsilon}^{k}\right\}, \varepsilon \in\left(-\varepsilon_{0}, \varepsilon_{0}\right), \varepsilon_{0}>0$, поверхности $F^{k}$, заданную уравнениями

$$
y^{\alpha}=f_{\varepsilon}^{\alpha}\left(x^{1}, x^{2}, \ldots, x^{k}\right)
$$

где $f_{\varepsilon}^{\alpha}-C^{2}$-непрерьвно зависящие от параметра $\varepsilon$ функции; $f_{0}^{\alpha} \equiv f^{\alpha}$. Каждая величина $A$ на $F^{k}$ при деформации перейдет в величину $A_{\varepsilon}$ на $F_{\varepsilon}^{k}$. Величины

$$
\delta(A)=\left.\frac{d A_{\varepsilon}}{d \varepsilon}\right|_{\varepsilon=0} \quad \text { и } \quad \widetilde{\delta}(A)=\frac{\delta(A)}{A}
$$

при $A \neq 0$ назьвают, соответственно, вариацией и относительной вариацией величины $A$, а тензорное поле $z^{\alpha}=\delta\left(f^{\alpha}\right)-$ полем деформации поверхности $F^{k}$. Две деформации поверхности $F^{k}$ в $\mathbb{R}^{n}$ являются эквивалентными, если их поля деформаций совпадают. Каждый из классов эквивалентных деформаций назьвают бесконечно малой деформацией поверхности $F^{k}$. В дальнейшем будем считать, что $z^{\alpha} \in C^{3}$.

Работа вьполнена при финансовой поддержке Российского фонда фундаментальных исследований, грант № 95-01-00228а. 
Бесконечно малую деформацию поверхности $F^{k}$ назьвают конформной (коротко $C$-деформацией), если вариации углов между любыми двумя направлениями в каждой точке поверхности равны нулю.

Известно [1], что $C$-деформации рассматриваемой поверхности $F^{k}$, и только они, характеризуются тем свойством, что относительная вариация $\widetilde{\delta}\left(d_{x} s\right)$ элемента длины дуги кривой на $F^{k}$ является функцией класса $C^{2}$ на $F^{k}$.

Пусть $G^{2}$ - двумерная поверхность, проходящая через точку $x$ поверхности $F^{k}$ в направлении двумерной площади $\xi$ и лежащая на поверхности $F^{k}$. Обозначим через $d_{x} \sigma\left(G^{2}\right)$ элемент площади поверхности $G^{2}$ в точке $x$. Бесконечно малую деформацию поверхности $F^{k}$ назовем ареально рекуррентной (коротко $A R$-деформацией), если относительная вариация $\widetilde{\delta}\left(d_{x} \sigma\left(G^{2}\right)\right)$ является функцией класса $C^{2}$ на $F^{k}$, т.е. в каждой точке $x \in F^{k}$ относительная вариация $\widetilde{\delta}\left(d_{x} \sigma\left(G^{2}\right)\right)$ не зависит от выбора двумерной плошадки $\xi$ и двумерной поверхности $G^{2}$, проходящей через точку $x$ в направлении $\xi$. Отсюда следует, что каждая $A R$-деформация характеризуется рекуррентньм соотношением:

$$
\delta\left(d_{x} \sigma\left(G^{2}\right)\right)=\varphi(x) d_{x} \sigma\left(G^{2}\right) \quad \forall x \in F^{k},
$$

где $\varphi$ - некоторая функция на $F^{k}$ класса $C^{2}$, определяемая бесконечно малой деформацией.

В настоящей работе доказывается следующая теорема.

Теорема 1. Для того, чтобы бесконечно малая деформачия поверхности $F^{k}, k>2$, в $\mathbb{R}^{n}$ была $A R$-деформацией, необходимо и достаточно, чтобы она была C-деформачий.

Пусть $G^{l}-l$-мерная $(2 \leqslant l \leqslant k)$ поверхность, проходящая через точку $x$, $x \in F^{k}$, в направлении $l$-мерной площадки $\eta$ и лежащая на $F^{k}$. Обозначим через $d_{x} \sigma\left(G^{l}\right)$ элемент $l$-мерного объема поверхности $G^{l}$ в точке $x$. Из теоремы 1 вытекает следующее следствие.

СлЕДСТВИЕ. Относительная вариация $\widetilde{\delta}\left(d_{x} \sigma\left(G^{l}\right)\right)$ nри AR-деформации поверхности $F^{k}$ является функиией на $F^{k}$, т.е. не зависит от выбора $l$-мерной площадки $\eta$ и поверхности $G^{l}$, и имеет место формула

$$
\widetilde{\delta}\left(d_{x} \sigma\left(G^{l}\right)\right)=l \widetilde{\delta}\left(d_{x} s\right) \quad \forall x \in F^{k} .
$$

В качестве приложения теоремы 1 приведем следующий результат.

ТЕОрема 2. Пусть $S^{n-1}$ - гиперсфера (или ее часть) в евклидовом пространстве $E^{n}, n>3$. Тогда каждая функиия $\varphi$ класса $C^{2}$, заданная на $S^{n-1}$, порождает семейство, зависящее от 
$n(n+1) / 2$ параметров, AR-деформаций поверхности $S^{n-1}$ с вариацией әлемента площади двумерной поверхности, определяемой рекуррентным соотношением (1) с заданной функиией $\varphi$. Каждое поле $A R$-деформачии представляет сумму полей бесконечно малого движения и нормальной бесконечной малой деформации $S^{n-1}$ в $E^{n}$.

Условие $k>2$ в приведенных теоремах является существенным. В случае $k=2 A R$-деформации составляют более широкий класс деформаций по сравнению с классом $C$-деформаций; случай $k=2, \varphi \equiv 0$ хорошо изучен в [2]. Теорема 1 в предположении $\varphi \equiv 0$ для случая евклидова пространства $E^{n}$ доказана в [3]. Нормальные бесконечно малые деформации изучались в [4].

2. Вывод уравнений $A R$-деформаций $F^{k}$ в $\mathbb{R}^{n}$. Зададим поверхность $G^{2}$ на $F^{k}, k>2$, уравнениями

$$
x^{i}=x^{i}\left(u^{1}, u^{2}\right), \quad\left(u^{1}, u^{2}\right) \in D .
$$

Тогда элемент площади $d_{x} \sigma\left(G^{2}\right)$ поверхности $G^{2}$ в точке $x$ вычисляется по формуле

$$
d_{x} \sigma\left(G^{2}\right)=\left(\operatorname{det}\left\|G_{p q}\right\|\right)^{1 / 2} d u^{1} \wedge d u^{2},
$$

где $G_{p q}=g_{i j} \xi_{(p)(q)}^{i} \xi^{j}-$ метрический тензор поверхности $G^{2} ; g_{i j}=a_{\alpha \beta} f_{, i}^{\alpha} f_{, j}^{\beta}-$ метрический тензор поверхности $F^{k} ; \xi^{i}=\partial x^{i} / \partial u^{p}, p=1,2$.

При $A R$-деформации поверхности $F^{k}$ имеем

$$
\left.\frac{d}{d \varepsilon}\left(d_{x} \sigma\left(G^{2}\right)\right)\right|_{\varepsilon=0}=2 \varphi\left(d_{x} \sigma\left(G^{2}\right)\right)^{2}
$$

где $\varphi$ - некоторая функция на $F^{k}$.

Отсюда в силу формулы (3) имеем

$$
\lim _{\varepsilon \rightarrow 0} \frac{1}{\varepsilon}\left\{\operatorname{det}\left\|G_{p q}^{\varepsilon}\right\|-\operatorname{det}\left\|G_{p q}\right\|\right\}=2 \varphi\left(\operatorname{det}\left\|G_{p q}\right\|\right),
$$

где

$$
\begin{aligned}
G_{p q}^{\varepsilon} & =g_{i j}^{\varepsilon} \xi_{(p)(q)}^{i} \xi^{j} ; \\
g_{i j}^{\varepsilon} & =a_{\alpha \beta}^{\varepsilon} f_{\varepsilon, i}^{\alpha} f_{\varepsilon, j}^{\beta} ; \\
a_{\alpha \beta}^{\varepsilon} & =a_{\alpha \beta}\left(f_{\varepsilon}^{1}, f_{\varepsilon}^{2}, \ldots, f_{\varepsilon}^{n}\right) .
\end{aligned}
$$


Разлагая функции $a_{\alpha \beta}^{\varepsilon}, f_{\varepsilon}^{\alpha}$ по степеням малого параметра $\varepsilon$, находим

$$
\begin{aligned}
g_{i j}^{\varepsilon}= & a_{\alpha \beta} f_{, i}^{\alpha} f_{, j}^{\beta} \\
& +\varepsilon\left[\partial_{\gamma} a_{\alpha \beta} z^{\gamma} f_{, i}^{\alpha} f_{, j}^{\beta}+a_{\alpha \beta} z_{, j}^{\alpha} f_{, i}^{\beta}+a_{\alpha \beta} z_{, i}^{\alpha} f_{, j}^{\beta}\right]+o(\varepsilon),
\end{aligned}
$$

где $o(\varepsilon)$ обозначают члены более высокого порядка малости по $\varepsilon$ при $\varepsilon \rightarrow 0$; $a_{\alpha \beta}, \partial_{\gamma} a_{\alpha \beta}$ вычислены в точках поверхности $F^{k}$.

Полагая

$$
\stackrel{*}{\nabla}_{i} z^{\alpha}=\partial_{i} z^{\alpha}+\Gamma_{\beta \gamma}^{\alpha} f_{, i}^{\beta} z^{\gamma},
$$

где $\Gamma_{\beta \gamma}^{\alpha}-$ символы Кристоффеля пространства $\mathbb{R}^{n}$, запишем

$$
g_{i j}^{\varepsilon}=g_{i j}+\varepsilon \delta g_{i j}+o(\varepsilon),
$$

где

$$
\delta g_{i j}=a_{\alpha \beta} \stackrel{*}{\nabla}_{(i} z^{\alpha} f_{, j)}^{\beta} .
$$

Используя полученное соотношение, запишем уравнение (4) в виде:

$$
D_{i j, k l} \xi^{i} \xi^{j} \xi^{k} \xi^{l}=0,
$$

где

$$
\text { (1) (2) (1) (2) }
$$

$$
D_{i j, k l}=\left(g_{i k} \delta g_{j l}+g_{j l} \delta g_{i k}-2 \varphi g_{i k} g_{j l}\right)[i, j]
$$

здесь знак $[i, j]$ означает альтернирование по индексам $i, j$.

Так как при $A R$-деформации поверхности $F^{k}$ соотношение (5) вьполняется для любых $\xi^{i}, \xi^{i}$, то коэффициенты многочлена (5) равны нулю, т.e.

$$
D_{i j, k l}+D_{k j, i l}+D_{i l, k j}+D_{k l, i j}=0 .
$$

Используя свойства символа $D_{i j, k l}$, известным приемом [1], отсюда находим

$$
D_{i j, k l}=0 \text {. }
$$

Умножая уравнение (7) на $g^{j l}$ и суммируя по индексам $j, l$, получаем

$$
g_{i k} g^{j l} \delta g_{j l}+(k-2) \delta g_{i k}=2 \varphi(k-1) g_{i k} .
$$

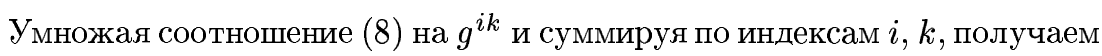

$$
g^{i j} \delta g_{i j}=k \varphi .
$$

Подставляя значение $\varphi$ из формулы (9) в формулу (7), получим уравнения $A R$-деформаций поверхности $F^{k}$ относительно искомого тензорного поля деформации $z^{\alpha}$ :

$$
\begin{aligned}
\left(k a_{\alpha \beta} \stackrel{*}{\nabla}_{(l} z^{\alpha} f_{, j)}^{\beta} g_{i r}+k a_{\alpha \beta} \stackrel{*}{\nabla}_{(i} z^{\alpha} f_{, r)}^{\beta}\right. & \\
& \left.-2 a_{\alpha \beta} \stackrel{*}{\nabla}_{(s} z^{\alpha} f_{, t)}^{\beta} g^{s t} g_{i k} g_{j l}\right)[i, j]=0 .
\end{aligned}
$$


3. ДоКАЗАТЕЛЬСТво ТЕОРЕМЫ 1. Покажем, что соотношения (10) описьвают $C$-деформации поверхности $F^{k}$. Так как $C$-деформации поверхности $F^{k}$ характеризуются соотношением $\delta\left(d_{x} s^{2}\right)=2 \psi\left(d_{x} s\right)^{2}$, где $\psi$ - относительная вариация элемента длины дуги кривой на $F^{k}$, то для $C$-деформаций имеем:

$$
\lim _{\varepsilon \rightarrow 0} \frac{1}{\varepsilon}\left[a_{\alpha \beta}^{\varepsilon} f_{\varepsilon, i}^{\alpha} f_{\varepsilon, j}^{\beta} d x^{i} d x^{j}-a_{\alpha \beta} f_{, i}^{\alpha} f_{, j}^{\beta} d x^{i} d x^{j}\right]=2 \psi g_{i j} d x^{i} d x^{j} .
$$

Последнее соотношение вьполняется для любых $d x^{1}, \ldots, d x^{k}$, и поэтому оно эквивалентно уравнениям

$$
\delta g_{i k}=2 \psi g_{i k}
$$

Соотношения (11) описьвают $C$-деформации поверхности $F^{k}$ в $\mathbb{R}^{n}$.

Обратимся к уравнениям (8), (9). Подставляя соотношение (9) в формулу (8), находим $(k-2)\left(\delta g_{i j}-\varphi g_{i j}\right)=0$.

Учитывая, что $k>2$, из последнего соотношения следуют уравнения (11) при $\varphi=2 \psi$. Это означает, что каждая $A R$-деформация является $C$-деформацией, причем

$$
\widetilde{\delta}\left(d_{x} \sigma\left(G^{2}\right)\right)=2 \widetilde{\delta}\left(d_{x} s\right) .
$$

Этим также доказана формула (2) при $k=2$.

Обратное утверждение теоремы 1 очевидно. Всякая $C$-деформация поверхности $F^{k}$ с относительной вариацией $\widetilde{\delta}\left(d_{x} s\right)=\psi(x)$ линейного элемента $d_{x} s$ является $A R$-деформацией поверхности $F^{k}$ с относительной вариацией $\widetilde{\delta}\left(d_{x} \sigma\left(G^{2}\right)\right)=2 \psi$. В самом деле, для $C$-деформации имеем $\delta g_{i j}=2 \psi g_{i j}$; тогда

$$
\delta G_{p q}=\delta g_{i j} \xi^{i} \xi^{j}=2 \psi \delta G_{p q}
$$

$(p)(q)$

и потому

$$
\begin{aligned}
& \delta\left(d_{x} \sigma\left(G^{2}\right)\right)=\delta\left(\sqrt{\operatorname{det}\left\|G_{p q}\right\|} d u^{1} \wedge d u^{2}\right) \\
& \quad=\frac{1}{2}\left(\operatorname{det}\left\|G_{p q}\right\|\right)^{-1 / 2}\left(G_{11} \delta G_{22}-2 G_{12} \delta G_{12}+G_{22} \delta G_{11}\right) d u^{1} \wedge d u^{2} \\
& \quad=2 \psi \sqrt{\operatorname{det}\left\|G_{p q}\right\|} d u^{1} \wedge d u^{2}=2 \psi d_{x} \sigma\left(G^{2}\right) .
\end{aligned}
$$

Это означает, что рассматриваемая $C$-деформация является $A R$-деформацией поверхности $F^{k}$. Теорема 1 доказана. 
4. Докажем справедливость формулы (2) при $k>2$.

Пусть $l=k$. Подсчитаем вариацию элемента $k$-мерного объема поверхности $F^{k}$.

Имеем

$$
\delta\left(d_{x} \sigma\left(F^{k}\right)\right)=\delta\left(\sqrt{\operatorname{det}\left\|g_{i j}\right\|} d x^{1} \wedge d x^{2} \wedge \cdots \wedge d x^{k}\right)
$$

Используя формулу (9), находим

$$
\begin{aligned}
& \delta\left(\operatorname{det}\left\|g_{i j}\right\|\right)=\sum_{i}\left|\begin{array}{ccccc}
g_{11} & \ldots & \delta g_{1 i} & \ldots & g_{1 k} \\
\ldots \ldots & \ldots & \ldots & \ldots & \ldots \\
\ldots \ldots & \ldots & \ldots & \ldots & \ldots \\
g_{k 1} & \ldots & \delta g_{k i} & \ldots & g_{k k}
\end{array}\right| \\
& =\operatorname{det}\left\|g_{i j}\right\| g^{i j} \delta g_{i j}=\operatorname{det}\left\|g_{i j}\right\| k \varphi \text {. }
\end{aligned}
$$

Это соотношение означает, что

$$
\delta\left(\sqrt{\operatorname{det}\left\|g_{i j}\right\|}\right)=\frac{k \varphi}{2} \sqrt{\operatorname{det}\left\|g_{i j}\right\|} .
$$

Поэтому имеет место формула

$$
\delta\left(d_{x} \sigma\left(F^{k}\right)\right)=\frac{k \varphi}{2} d_{x} \sigma\left(F^{k}\right)
$$

где

$$
\varphi=\widetilde{\delta}\left(d_{x} \sigma\left(F^{2}\right)\right)=2 \widetilde{\delta}\left(d_{x} s\right)
$$

Этим доказана формула (2) при $l=k$.

Если $l<k$, то $A R$-деформация поверхности $F^{k}$ порождает $A R$-деформацию поверхности $G^{l}$, и поэтому будет вьполняться соотношение

$$
\delta\left(d_{x} \sigma\left(G^{l}\right)\right)=\frac{l}{2} \varphi(x) d_{x} \sigma\left(G^{l}\right)=l \widetilde{\delta}\left(d_{x} s\right) d_{x} \sigma\left(G^{l}\right)
$$

Формула (2) доказана. 
5. ДокАЗАТЕЛЬСтво теоремЫ 2 . Пусть $\left(x^{1}, x^{2}, \ldots, x^{n-1}\right)-$ координаты карты атласа на гиперсфере $S^{n-1}$ в $E^{n}$ с декартовыми координатами $\left(y^{1}, y^{2}, \ldots, y^{n}\right)$. Тогда уравнения $A R$-деформации, определяемой заданной на $S^{n-1}$ функцией $\varphi$, имеют вид

$$
a_{\alpha \beta} f_{\left.,{ }_{i}, j\right)}^{\alpha} z_{, j)}^{\beta}=\varphi g_{i j}
$$

где $z^{\beta}$ - искомое тензорное поле.

Положим $a_{i}=a_{\alpha \beta} z^{\alpha} f_{, i}^{\beta}$. Тогда, используя уравнения $(12)$, имеем

$$
a_{i, j}=a_{\alpha \beta} \xi^{\alpha} z^{\beta} b_{i j}+a_{\alpha \beta} z_{, j}^{\alpha} f_{, i}^{\beta}
$$

где $\xi^{\beta}$ - единичньй вектор нормали к $S^{n-1}, b_{i j}$ - тензор второй квадратичной формы $S^{n-1}$. Так как справедливы соотношения $b_{i j}=g_{i j} / R$, где $R=$ const, то уравнения $A R$-деформаций $S^{n-1}$ принимают вид:

$$
a_{(i, j)}=2 c b_{i j}+R \varphi b_{i j}
$$

где $c=a_{\alpha \beta} z^{\alpha} \xi^{\beta}$.

Систему (13) можно рассматривать как систему относительно искомых функций $a_{1}, a_{2}, \ldots, a_{n-1}, c+R \varphi / 2$. Тог да ее можно интерпретировать как систему уравнений бесконечно малых изгибаний $S^{n-1}$ в $E^{n}$. Как известно [1], $S^{n-1}$ жестка в $E^{n}$, и поэтому система (13) имеет точно $n(n+1) / 2$ решений, порождаемьх тривиальными бесконечно мальми изгибаниями $S^{n-1}$. Каждое из таких решений порождает решение $\left(a_{i}, c\right)$ системы $(13)$, что и доказьвает теорему 2.

Таганрогский государственный Поступило педагогический институт 30.06 .94

\section{СПИСОК ЦИТИРОВАННОЙ ЛИТЕРАТУРЫ}

[1] Рашевский П. К. Риманова геометрия. М.: Наука, 1964.

[2] Бескоровайная Л. Л. Канонические $A$-деформации, сохраняющие длины линий кривизны поверхности // Матем. сб. 1975. Т. 93. № 2. С. 163-176.

[3] Фоменко Л.П. $A^{2}$-деформации поверхностей $F^{k}$ в $E^{n} / /$ Сиб. матем. ж. 1991. Т. 32. № 5. С. 204; // Деп. ВИНИТИ. № 5543-В90.

[4] Chen B. Y., Yano K. On the theory of normal variations // J. Diff. Geom. 1978. V. 13. P. 1-10. 